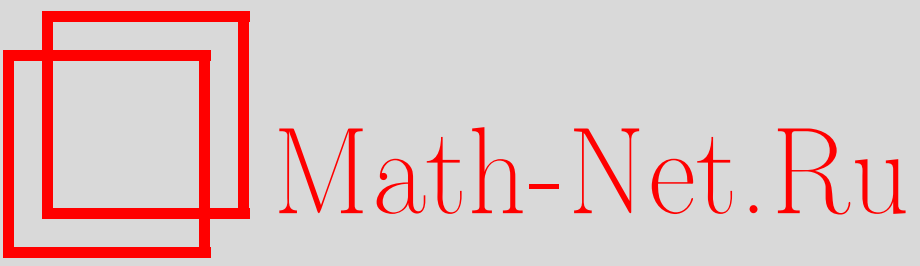

И. Б. Краснюк, Нелинейные граничные задачи для уравнения Больцмана: периодические решения и их бифуркации, ТМФ, 1997, том 110, номер 2, 323-333

DOI: https://doi.org/10.4213/tmf971

Использование Общероссийского математического портала Math-Net.Ru подразумевает, что вы прочитали и согласны с пользовательским соглашением

http://www.mathnet.ru/rus/agreement

Параметры загрузки:

IP: 18.207 .199 .55

26 апреля 2023 г., 14:12:44 


\section{НЕЛИНЕЙНЫЕ ГРАНИЧНЫЕ ЗАДАЧИ ДЛЯ УРАВНЕНИЯ БОЛЬЦМАНА: ПЕРИОДИЧЕСКИЕ РЕШЕНИЯ И ИХ БИФУРКАЦИИ}

Рассматривается проблема постановки нелинейных краевых условий для кинетического уравнения Больцмана. На этой основе обсуждается возможность образования в газах асимптотически периодических колебаний релаксационного или предтурбулентного типов.

1. Уравнение Больцмана для функции распределения $f(X, \xi, t)$ молекул одноатомного газа по координате $X$ и скорости $\xi$ в момент времени $t$ представляет собой интегродифференциальное уравнение

$$
\frac{\partial f}{\partial t}+\xi \frac{\partial f}{\partial X}+X \frac{\partial f}{\partial \xi}=Q(f, f)
$$

где $Q($,$) - билинейный оператор, называемый оператором столкновений, а X$ - действуюшая на молекулу внешняя сила, отнесенная к единице массы.

Отметим, что при общем подходе в большинстве случаев рассматриваются простые краевые условия для уравнения Больцмана. Считается, что газ находится в неограниченной области, или же, если область ограничена, то на ее границе заданы условия периодичности или зеркального отражения молекул (ниже будет показано в качестве частного случая, что такие граничные условия, если они физичны, не приводят к образованию структур). Как отмечено в [1, с. 133]: "В случае течения газа около твердого тела или внутри области, ограниченной одним или несколькими твердыми телами, эти упрощения недопустимы и тогда краевые условия описывают взаимодействие молекул газа с твердыми стенками, т.е. взаимодействие, следствием которого являются сила, действующая на тело со стороны газа (лобовое сопротивление и подъемная сила), и теплопередача между газом и границей твердого тела. К сожалению, как теоретическая, так и экспериментальная информация о взаимодействии газ - поверхность довольно ограничена и зачастую сводится к обшим утверждениям либо к построению интуитивных моделей; между тем рассматриваемый метод требует полного знания нелинейного обмена энергией, т.е. знания $F$ - функции взаимодействия".

В этой статье в общих чертах представлена "теоретическая информация" о корректной постановке краевых условий и показано, как для уравнений Больцмана в “нулевом

*НПО “Физика-Солнце” АН РУз 


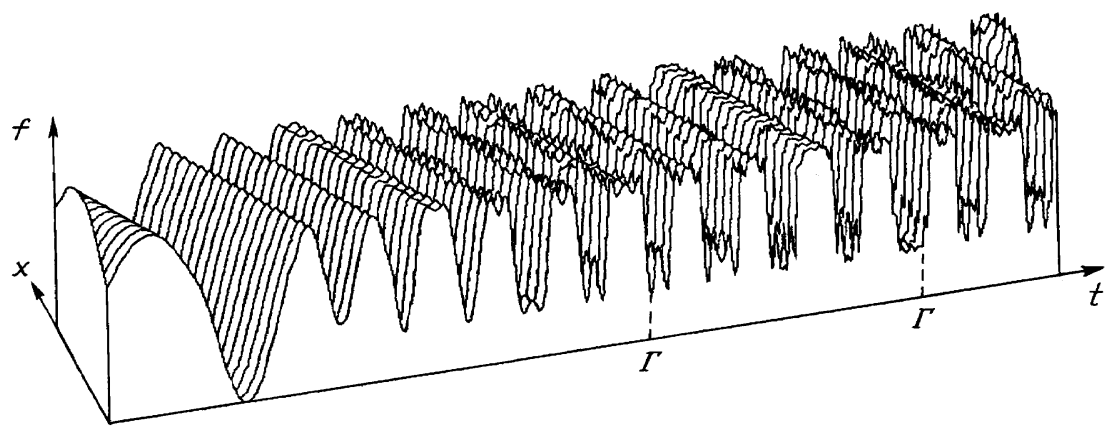

Рис. 1. Предельные распределения релаксационного типа.

приближении" по граничным условиям на качественном уровне понимания предсказывать возможность образования асимптотически периодических колебаний релаксационного (рис. 1) или предтурбулентного (рис. 2) типов по терминологии работы [2]. Напомним, что колебаниями релаксационного типа называются такие асимптотически периодические распределения, при которых число точек разрьвов Г на периоде предельного решения конечно. Если множество Г счетно на периоде (и, следовательно, имеет некоторую точку сгушения или несколько таких точек), то мы имеем дело с колебаниями предтурбулентного типа. Под точками разрывов предельного решения мы понимаем точки множества $\Gamma$, в которых производная по времени при $t \rightarrow \infty$ сколь угодно велика, хотя само предельное решение является гладким и даже сколь угодно гладким при достаточно гладких начальных распределениях краевой задачи. Более сложная формальная топологическая природа образования таких колебаний с точки зрения теории одномерных отображений подробно рассматривалась в работе [2]. Там же дано математическое определение упорядоченных структур. Однако мы будем использовать этот термин лиш как “эквивалентное” название для колебаний релаксационного или предтурбулентного типов. Предлагаемый нами метод сушественно опирается на нелинейность краевых условий.

Основное понятие, в терминах которого формулируются краевые условия, - плотность вероятности $R\left(\xi^{\prime} \rightarrow \xi ; X, t ; \tau\right)$ того, что молекула, достигаюшая поверхности со скоростью между $\xi^{\prime}$ и $\xi^{\prime}+d \xi^{\prime}$ в точке $X$ в момент времени $t$, вылетит практически в той же точке (что, вообще говоря, не очевидно!) со скоростью между $\xi$ и $\xi+d \xi$ в момент времени $(t+\tau)$, где $\tau$ - время адсорбции или задержки.

Отметим, что время задержки может являться (но не всегда) бифуркационным параметром [3-8], приводяшим в случае нелинейных граничных условий к асимптотически периодической функции распределения $f(\cdot)$ с периодом, пропорциональным времени $T \sim$ const $\cdot \tau$, где постоянная зависит в общем случае от параметров задачи (плотности газа, коэффициента теплопроводности и т.д.).

Если $R$ известна, то легко записать граничное условие [1, с. 133]

$$
|\xi \cdot n| f(x, \xi, t)=\int_{0}^{\infty} d \tau \int_{\xi^{\prime} \cdot n<0} R\left(\xi^{\prime} \rightarrow \xi ; x, t ; \tau\right) f\left(x, \xi^{\prime}, t-\tau\right)\left|\xi^{\prime} \cdot n\right| d \xi^{\prime},
$$

где $\xi \cdot n>0, x \in \partial \Omega, n$ - единичный вектор нормали к поверхности. 


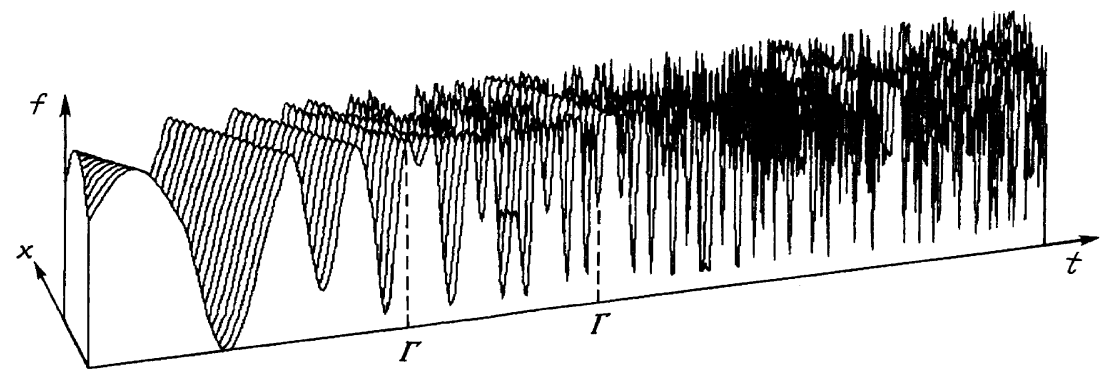

Рис. 2. Предельные распределения предтурбулентного типа.

Пусть вьполняется неравенство

$$
n \sigma_{0}^{2} \cdot \bar{v} \bar{\tau} \ll 1
$$

где $n$ - плотность числа частиц, $\sigma_{0}$ - эффективный радиус взаимодействия газа с поверхностью, $\bar{v}$ - средняя нормальная составляющая скорости падающих на поверхность молекул, $\bar{\tau}$ - эффективное время адсорбции. Тогда можно считать, что $R\left(\xi^{\prime} \rightarrow \xi ; x, t ; \tau\right)$ не зависит от функции распределения $f\left(X, \xi^{\prime}, t\right)$. Это приводит, в свою очередь, к возможности вычисления ядра рассеяния в предположении, что на поверхность налетает одна молекула с заданной скоростью $\xi^{\prime}$. В этом случае предельная плотность $f$ при $t \rightarrow \infty$ стремится либо к нулю, либо к бесконечности, причем случай зеркального отражения структурно-неустойчив [4], т.е. он допускает сушествование асимптотически периодических решений, которые, однако, "рассыпаются" при сколь угодно малых регулярных возмущениях [5].

Таким образом, мы получаем необходимое (но не достаточное) условие возникновения упорядоченных структур

$$
n \sigma_{0}^{2} \bar{v} \tau>1
$$

Если к тому же $\tau$ мало по сравнению с любым характерным временем, отвечающим эволюции функции $f$, то в граничных условиях можно положить $\tau=0$; в этом случае возможность образования колебательных режимов для таких краевых задач нам неизвестна. Дело в том, что условие $\tau>0$ “помогает" формированию локализованного решения для плотности $f$ "волнового пакета", для которого в высокочастотном приближении удается доказать [6], что граничные условия порождают колебательные режимы для линеаризованных уравнений Больцмана аналогично тому, как это можно сделать, например, при исследовании нелинейно связанных обобщенных уравнений Шредингеpa $[9]$.

Если граница возврашает все молекулы газа (т.е. она не пористая и не адсорбируюшая), то полная вероятность того, что падающая молекула вылетит обратно с некоторой скоростью $\xi$, равна единище:

$$
\int_{\xi \cdot n>0} R\left(\xi^{\prime} \rightarrow \xi ; x, t\right) d \xi=1
$$


Кроме того, ядро неотрицательно, т.е.

$$
R\left(\xi^{\prime} \rightarrow \xi ; x, t\right) \geqslant 0
$$

Следуюшее свойство ядра представимо соотношением

$$
\left|\xi^{\prime} \cdot n^{\prime}\right| f_{\omega}\left(\xi^{\prime}\right) R\left(\xi^{\prime} \rightarrow \xi\right)=|\xi \cdot n| R\left(-\xi \rightarrow-\xi^{\prime}\right) f_{\omega}(\xi),
$$

которое может быть названо законом взаимности или принципом детального баланса. Следствием последнего соотношения является то, что функция $f_{\omega}$ (максвелловская) удовлетворяет граничным условиям

$$
\int_{\xi^{\prime} \cdot n<0}\left|\xi^{\prime} \cdot n\right| f_{\omega}\left(\xi^{\prime}\right) R\left(\xi^{\prime} \rightarrow \xi\right) d \xi^{\prime}=|\xi \cdot n| f_{\omega}(\xi), \quad \xi \cdot n>0,
$$

что нетрудно показать, если проинтегрировать равенство (3) по $\xi^{\prime}$ и воспользоваться условием (2).

Пусть, например, $f^{ \pm}$- плотности распределения молекул одноатомного газа, которые имеют скорости $( \pm \xi)$, соответственно, причем рассматривается сильно разреженньй газ; поэтому в нулевом приближении можно считать, что $Q(\cdot, \cdot)=0$ и действующие на молекулы внешние силы также равны нулю. Тогда уравнение Больцмана идеального газа для плотностей распределений молекул по скоростям $( \pm \xi)$ можно записать в виде

$$
\begin{aligned}
\frac{\partial f^{+}}{\partial t}+\xi \frac{\partial f^{+}}{\partial x} & =0, \\
\frac{\partial f^{-}}{\partial t}+\xi^{\prime} \frac{\partial f^{-}}{\partial x} & =0 .
\end{aligned}
$$

Рассмотрим область $\Pi=\{X, t: 0 \leqslant X \leqslant 1, t \geqslant 0\}$ и поставим следуюшие граничные условия:

$$
\begin{aligned}
\xi \cdot n f^{+}(\xi, 1) R_{1}^{1}(\xi \rightarrow-\xi) & =-\xi \cdot(-n) f^{-}(-\xi, 1) R_{2}^{1}(-\xi \rightarrow \xi), \\
-\xi \cdot n f^{-}(-\xi, 0) R_{2}^{0}(-\xi \rightarrow \xi) & =\xi \cdot(-n) f^{+}(\xi, 0) R_{1}^{0}(\xi \rightarrow-\xi),
\end{aligned}
$$

и начальные условия:

$$
f^{+}(\xi, x, 0)=h_{1}(x), \quad f^{-}(-\xi, x, 0)=h_{2}(x), \quad 0 \leqslant x \leqslant 1 .
$$

Здесь $R_{1}^{1}$ - плотность вероятности того, что молекула, падаюшая на поверхность $X=1$ со скоростью $\xi$, отразится от этой поверхности со скоростью $-\xi$; остальные коэффициенты имеют тот же смысл. Граничные условия (5) отражают по существу закон взаимности (3). Конечно, строго следовало бы записывать граничные условия $\left(3^{\prime}\right)$, где $R=R_{1}^{1} \backslash R_{2}^{1}$, однако такая задача слишком сложна для детального исследования.

Поскольку плотности вероятностей на левой и правой границах могут быть, вообще говоря, различными, то, не ограничивая общности, можно считать $R_{1}^{0}=R_{2}^{0}$. Если дополнительно принять, что $R_{1}^{1}=R_{2}^{1}$, то задача (4)-(6) для любых начальных распределений $h_{1}$ и $h_{2}$ имеет асимптотически (при $t \rightarrow \infty$ ) 2-периодические распределения плотностей вида $f(t+X / \xi)$ и $f(t+X /(-\xi))$, которые являются структурно-неустойчивыми [2]: при $R>1$ плотности уходят на бесконечность, а при $R<1-$ к нулю. 
Единственный урок, который отсюда можно извлечь - это предположить формальное выполнение граничного условия при $x=1$ :

$$
f^{+}=R\left(f^{+}, f^{-}\right) f^{-},
$$

где плотности вероятностей зависят от неизвестных плотностей распределений газа по скоростям $\xi$ и $-\xi$, соответственно.

Как отмечено в [1, с. 136], обшие рассуждения могут привести лишь к условиям типа взаимности. Там же утверждается, что по этой причине, вообще говоря, необходимо построить физическую модель поверхности и попытаться определить соответствующее ядро $R\left(\xi^{\prime} \rightarrow \xi\right)$ или построить математическую модель ядра, удовлетворяюшую требованию относительной простоты. Граничное условие $\left(5^{\prime}\right)$ является формальной моделью такого "ядра".

Конечно, закон взаимности не имеет реального отношения к образованию упорядоченных структур и приведен здесь лишь для иллюстрации того, что когда нелинейное граничное условие $\left(5^{\prime}\right)$ вырождается в соотношение детального равновесия, то в модели (4)-(6) ничего интересного не происходит. Реальное отношение к граничному условию имеет не закон взаимности, а сильная нелинейность ядра рассеяния $R$ в граничном условии (1), поскольку только в этом случае можно говорить о возможности образования упорядоченных структур.

Отметим, что вместо двухкомпонентной модели (4) идеального газа можно рассмотреть счетный набор по скоростям. Тогда уравнение (4) запишется в матричной форме с граничными условиями вида (5), где $f$ - вектор из $n$ компонент, а $R$ - аналог матрищы рассеяния размера $n \times n$. Из результатов $[1,8]$ нетрудно показать, что если все собственные значения матрицы $R$ вешественны и меньше по абсолютной величине 1 , то все компоненты плотностей по $n$ скоростям сходятся к нулю, а при абсолютной величине больше 1 - к бесконечности. Формальная ситуация, когда $f$ - нелинейный функционал, рассмотрена в $[2,10]$, в этом случае можно говорить, что каждая компонента плотности имеет колебания релаксационного или предтурбулентного типов. Конечно, вопрос о предельном переходе $n \rightarrow \infty$ остается открытым.

2. Известно [1, с. 139], что при решении уравнений Больцмана в реальных неравновесных ситуациях мы вынуж дены пользоваться приближенными методами, в частности методами теории возмущений. По этой причине мы должны обратиться к линеаризованному уравнению вида (4). Первый шаг здесь состоит в исследовании порядков различных величин, входящих в уравнение Больцмана.

Обозначим через $\tau, d$ и $\bar{\xi}$ соответственно характерные времена, длину и скорость молекул. Тогда имеем

$$
\frac{\partial f}{\partial t} \sim \tau^{-1} f, \quad \xi \frac{\partial f}{\partial X} \sim \bar{\xi} d^{-1} f, \quad Q(f, f) \sim n \bar{\xi} \sigma^{2} f,
$$

здесь $n=\rho / m$ - среднее число молекул в единице объема, $\sigma$ - диаметр молекул (или радиус действия межмолекулярных сил). В соответствии с определением средней длины свободного пробега молекул $l$ мы будем иметь

$$
Q(f, f) \sim \bar{\xi} l^{-1} f .
$$


В этой связи следует отметить [1], что понятие среднего свободного пробега может быть строго определено только в случае твердых сфер или обрезанных потенциалов $(\sigma<\infty)$; для дальнодействующих потенциалов $(\sigma \mapsto \infty)$, т.е. когда молекулы взаимодействуют всюду, длина свободного пробега, вообще говоря, равна нулю. Однако несмотря на это понятие средней длины свободного пробега можно сохранить как инструмент для оценки правой части уравнения Больцмана, т.е. длина свободного пробега определяется скорее качественно, чем количественно. Интересно сравнить такое (качественное) понимание проблемы с аналогичным подходом в задачах теплопереноса [3].

На примере краевой задачи для системы (4) можно показать [3], что параметр $\mu=$ $n \bar{\xi} \sigma^{2} f$ является диссипативным, т.е. что можно подобрать такие граничные условия, при которых для модели “точечных" потенщиалов $(\sigma \ll 1)$ мы получаем асимптотически периодические распределения $f^{+}, f^{-}$, близкие к кусочно-постоянным периодическим плотностям [6]. В случае же $\sigma \gg 1$ (дальнодействующие потенциалы) плотности $f^{+}$, $f^{-}$почти всюду по сечению образца стремятся к нулю при $t \rightarrow \infty$ за исключением конечного или счетного числа “ $\delta$-всплесков”, которые возникают за счет специального выбора нелинейных краевых условий [5].

Этому определению можно придать и количественный смысл, если вспомнить, что коэффициент вязкости $\mu$ пропорционален $l$ :

$$
l=\mu(\pi R T / 2)^{1 / 2} / p=\mu[\pi /(2 R T)] \rho^{1 / 2},
$$

где $p=\rho R T$ - давление. Отсюда (при разумных скоростях $\xi$ ) получаем, что при $T \ll$ $(\mu / \rho)^{2}$ плотность $f$ близка асимптотически кусочно-постоянной функции с амплитудой затухания, пропорциональной $\exp (\bar{\xi} / l) x[5]$.

Величину $\theta=\xi^{-1} l$ естественно считать по определению средним временем свободного пробега, а обратную к ней величину $\xi l^{-1}-$ мерой частоты столкновений. Таким образом, когда мера частоты столкновений велика, на гранище области возникает пограничный слой [6].

Из указанных выше соотношений следует, что в уравнении Больцмана сушественную роль должны играть два основных безразмерных параметра: $(\theta / \tau)$ и $(l / d)$. Обозначим $\mathrm{K}=l / d$ - число Кнудсена, где $0<\mathrm{K}<\infty$. Случай $\mathrm{K} \rightarrow 0$ соответствует плотному газу (в котором возможно сушествование сингулярного пограничного слоя), а случай $\mathrm{K} \rightarrow \infty-$ свободномолекулярному течению, в котором можно пренебречь взаимодействием молекул.

Заметим, что

$$
\frac{\theta}{\tau}=\frac{\xi^{-1} l}{\tau}=\left(\frac{d}{\bar{\xi} \tau}\right) \mathrm{K}=\mathrm{Sh} \mathrm{K},
$$

где $\mathrm{Sh}=d /(\bar{\xi} \tau)-$ число Струхаля, которое используется в гидродинамических задачах ( $\bar{\xi}$ обычно имеет порядок скорости звука). Как отмечено в [1, с. 144], указанные параметры имеют смысл лиш для задач, в которых, кроме длины свободного пробега, есть лишь одна характерная длина [1], в противном случае надо рассматривать несколько чисел Кнудсена.

Интересно отметить, что можно изучать класс задач для систем дискретных скоростей (или даже не обязательно гидродинамических задач), для которых (например, для двух систем) имеют место соотношения [10]

$$
\frac{\mathrm{K}_{1}}{\mathrm{~K}_{2}}=\left(\frac{l_{1}}{l_{2}}\right) /\left(\frac{d_{1}}{d_{2}}\right)=\frac{p}{q}=\text { рац. число. }
$$


Физического объяснения этому соотношению пока нет.

Можно показать [10], что если вязкость $\mu \gg 1$, то не следует ожидать появления пограничного слоя.

3. Обычно рассматриваются два вида разложений: для $\mathrm{K} \rightarrow 0$ и для $\mathrm{K} \rightarrow \infty$. Из формальных результатов [6] следует, что при $\mathrm{K} \gg 1$ достаточно ограничиться методами стандартной теории возмущений по малому параметру $\varepsilon=\mathrm{K}^{-1}$. При этом граничные условия играют роль "стабилизатора" амплитуд " $f$-возмушений" [5], и решение при $t \rightarrow \infty$ выходит на устойчивый периодический режим.

При $\mathrm{K} \gg 1$ мы получаем сингулярно возмушенное уравнение Больцмана (аналогичные уравнения возникают в задачах теплопереноса [3], химической кинетики [8], лазерной физики [6] и т.д., этой аналогией мы и будем всюду в дальнейшем пользоваться), в этом случае необходим специальный выбор начальных (и частично граничных) условий [5]. При этом сходимость плотностей $f^{\varepsilon} \Rightarrow f$ при $\varepsilon \rightarrow 0$ равномерна (в обычной метрике) почти всюду, за исключением некоторого множества точек разрывов $\Gamma$, которое определяется граничными условиями (см. рис. 1) [11].

При $\mathrm{K} \ll 1$ сходимости по сингулярному параметру $\varepsilon$, вообше говоря, не сушествует при $\hat{\varepsilon}=\mathrm{K}$ для решений релаксационного типа, хотя предельное решение и устойчиво к возмушениям по параметру в окрестности каждого фиксированного $\mathrm{K}_{0}$ [11].

Предположим, что число Кнудсена K мало, а число Струхаля $\mathrm{Sh}$ - величина порядка единицы. Полагая, что число Кнудсена $\mathrm{K}=\varepsilon$, мы получаем уравнение

$$
\varepsilon\left(\frac{\partial f}{\partial t}+\xi \frac{\partial f}{\partial X}\right)=Q(f, f)
$$

Будем искать решение в виде ряда

$$
f=\sum_{n=0}^{\infty} \varepsilon^{n} \cdot f_{n}
$$

которьй теряет равномерную сходимость в окрестности множества Г-точек разрывов в тех областях, где профили плотности, скорости и температуры становятся очень крутыми при $\varepsilon \rightarrow 0 ;$ к таким слоям относятся окрестности границ (пограничные слои), начальный промежуток времени (начальньй слой) и ударные волны (ударные слои). В $[1$, с. 149$]$ отмечается, что первые два типа слоев сушествуют и для линеаризованного уравнения Больцмана, в то время как ударньй слой - это область больших градиентов и его сушествование связано с нелинейностью уравнения Больцмана.

Как показывают результаты [6], ударные слои могут существовать и для линеаризованного уравнения Больцмана с нелинейными (не обязательно интегральными) граничными условиями; причем, если ядро $R\left(\xi^{\prime} \rightarrow \xi\right)$ зависит от плотности $f\left(x, \xi^{\prime}, t\right)$ нелинейно (достаточно квадратичной нелинейности), то возможно появление конечного числа, счетного числа и даже несчетного числа ударных слоев. В последнем случае множество точек разрывов $Г$ имеет структуру канторова множества, а само предельное распределение $f$ имеет вид "канторовой гребенки" (см. рис. 2).

Указанные выше решения иногда называют нормальными. Если решения не являются нормальными, то можно использовать другие представления, обобшаюшие методы 
усреднения Боголюбова-Митропольского $[12,13]$, дополняюшие метод ВКБ $[14,15]$ и т.д. Тогда предыдущее разложение можно представить в виде

$$
f\left(x, t, \frac{S}{h}\right)=\sum_{n=0}^{\infty} \varepsilon^{n} f_{n}\left(x, t, \frac{S}{h}\right)
$$

где $S$ - вешественная фаза, удовлетворяющая уравнению Гамильтона-Якоби; $h=\mathrm{K}-$ число Кнудсена.

Отметим, что полученные выше (формально) рациональные отношения чисел Кнудсена [10] для некоторых специальных краевых задач, возможно, найдут объяснение в терминах когерентного согласования фаз:

$$
\frac{S_{1}}{S_{2}}=\frac{p}{q}=\text { рац. число, }
$$

что, вообше говоря, более правдоподобно, поскольку ясно, что в этом случае должна формироваться когерентная волна [6].

ПримеР. В случае свободномолекулярного течения, когда $\mathrm{K} \rightarrow \infty$, уравнение Больцмана (в отсутствие внешних сил) имеет вид

$$
\frac{\partial f}{\partial t}+\xi \frac{\partial f}{\partial X}=0
$$

главную роль здесь играет взаимодействие молекул с твердыми стенками.

Рассмотрим теперь задачу, когда взаимодействие с границей отсутствует, на примере уравнений [16]

$$
\begin{gathered}
\frac{\partial u}{\partial t}+\frac{\partial u}{\partial x}=v^{2}-u w \\
\frac{\partial v}{\partial t}=u w-v^{2} \\
\frac{\partial w}{\partial t}-\frac{\partial w}{\partial x}=v^{2}-u w
\end{gathered}
$$

которые представляют собой так называемую упрошенную модель Бродуэлла [17].

Точнее, мы ограничимся еше более простой моделью системы

$$
\begin{aligned}
\frac{\partial u}{\partial t}+\frac{\partial u}{\partial x} & =\mu^{2}-u w \\
\frac{\partial w}{\partial t}+\frac{\partial w}{\partial x} & =\rho^{2}-\mu u
\end{aligned}
$$

с граничными условиями

$$
\frac{\partial u}{\partial x}=\frac{\partial w}{\partial x}=0 \text { при } x=0, \quad u+w-\mu^{2}=0 \text { при } x=1
$$

и некоторыми начальными условиями. 
В модели [16] дано 3 вешества, распределенных на интервале $0<x<1$, которые вступают в реакцию со скоростями $(1,0,-1)$. Удобно в системе $(7)$ вместо второго уравнения рассмотреть уравнение

$$
\frac{\partial v}{\partial t}=u+w-v^{2},
$$

где предполагается, что концентрация вешества $v$ не изменяется со временем, т.е. $(\partial v / \partial t) \equiv 0$ при всех $t \geqslant 0$. Тогда, поскольку $v$ не зависит от $X$, а функции $u, w$ зависят, то из (10) нетрудно получить краевое условие (9) при $x=1$. Такая форма граничного условия выбрана лишь из соображений удобства для иллюстрации редукции задачи $(8),(9)$ к разностному уравнению. Вообше говоря, можно рассматривать обычные граничные условия вида $\partial w / \partial x=S(w)$, где $S(\cdot)$ - некоторая функция [18].

Назовем кинетическую систему положительной, если для всех неотрицательных начальных условий $z_{i}(0, x) \geqslant 0$ выполнены неравенства $z_{i}(t, x) \geqslant 0$ для всех $t \geqslant 0$. Условия, при которых это определение содержательно, получены для так называемых Б-систем (больцмановских систем) Веденяпиным [17]. Из $[18,19]$ следует, что при выполнении условий, близких к условиям из [17], общее решение краевых задач для кинетических систем представимо при больших временах в виде промежуточных асимптотик [2]:

$$
f(t, x, \xi)=p(t+x / \xi, \xi),
$$

где $\xi= \pm 1$, а $p$-произвольная функция. Последнее соотношение означает, что функция $p$ не изменяется вдоль прямолинейных траекторий молекул $(x \pm \xi t)=X_{ \pm}$; этим фактом мы воспользуемся в дальнейшем.

Из [18] следует, что граничное условие (9) при $x=0$ можно свести к функциональному, если скорость увеличения $i$-го вешества $F_{i}\left(z_{1}, z_{2}\right)$ удовлетворяет так называемому условию вполне интегрируемости [2]. На примере задачи (8), (9) это означает следующее: поскольку рассматриваются гладкие решения, то уравнения (8) должны вьполняться не только почти везде внутри области, но и на границе. Тогда при $x=0$ с учетом граничных условий (9) и уравнений (8) мы получаем новое краевое условие

$$
\frac{\partial u}{\partial t}=\mu^{2}-u w, \quad \frac{\partial w}{\partial t}=\rho^{2}-\mu u .
$$

Очевидно, что система (11) имеет общий интеграл

$$
\rho^{2} u-\frac{\mu}{2} u=\mu^{2} w-\frac{1}{2} u w^{2} .
$$

Если кинетическая система положительно определена, то можно считать, что выполнено условие $u \neq 0$ почти везде, и записать при $\rho, \mu \ll 1$ (это требование не приншипиально и необходимо лиш для удобства изложения) в точке $x=0$ приближенные соотношения

$$
\frac{\mu}{2} u^{2}=\frac{1}{2} u w^{2} \text { или } \mu u=w^{2} .
$$

То что такая процедура корректна и допускает решения, близкие к решениям исходной задачи, доказано в $[2,18,19]$. 
Тогда в силу постоянства функций $u$ и $w$ (при больших временах) вдоль характеристик $d x / d t=1$ и $d x / d t=-1$ мы можем (с учетом функциональных условий (12) и (9)) получить цепочку соотношений [2]

$$
\begin{aligned}
u(1, t+1) & =u(0, t)=\frac{1}{\mu} w^{2}(0, t)=\frac{1}{\mu} w(1, t-1)=\frac{1}{\mu}(-u(1, t-1)+\mu)^{2}= \\
& =\left(\frac{1}{\mu} u^{2}(1, t-1)-2 u(1, t-1)+\mu\right)
\end{aligned}
$$

Итак, краевая задача свелась к исследованию итераций траекторий разностного уравнения с непрерывным временем

$$
u(1, t+1)=\frac{1}{\mu} u^{2}(1, t-1)-2 u(1, t-1)+\mu
$$

с некоторой начальной функцией на интервале [0, 1]. Известно [2], что существуют значения $\mu$ из ограниченного интервала, при которых решения сходятся к кусочно-постоянным $2^{N}$-периодическим функциям, принимаюшим значения из множества притягивающих неподвижных точек квадратичного отображения $u \mapsto \frac{1}{\mu} u^{2}-2 u+\mu$, а $N$ - наименьшее общее кратное периодов притягивающих циклов этого отображения [2].

ЗАмЕчАниЕ. Для системы Карлемана

$$
\frac{\partial z_{1}}{\partial t}+\frac{\partial z_{1}}{\partial x}=z_{2}^{2}-z_{1}^{2}, \quad \frac{\partial z_{2}}{\partial t}-\frac{\partial z_{2}}{\partial x}=z_{1}^{2}-z_{2}^{2}
$$

с нулевыми граничными условиями Неймана мы приходим при больших временах к исследованию уравнения

$$
z^{2}(1, t+1)=z^{2}(1, t-1)
$$

которое имеет семейство 2-периодических решений, однако все они структурно-неустойчивы, т.е. разрушаются при сколь угодно малых регулярных возмушениях.

Если граничные условия нелинейны, то решения краевой задачи для уравнений (13) асимптотически устойчивы. В то же время, если первое из уравнений (13) заменить, например, уравнением

$$
\frac{\partial z_{1}}{\partial t}+\frac{\partial z_{1}}{\partial x}=z_{2}^{2}-\left(z_{1}+\varepsilon\right)
$$

где $\varepsilon>0$ - некоторое число, то можно получить предельные распределения релаксационного или предтурбулентного типов даже для однородных краевых условий. 


\section{Список литературы}

[1] Уравнение Больцмана и его приложения. М.: Мир, 1986.

[2] А.Н. Шарковский, Ю. Л. Майстренко, Е. Ю. Романенко. Разностные уравнения и их приложения. Киев: Наукова думка, 1986.

[3] И. Б. Краснюк. ИФЖ. 1994. Т. 67. № 1, 2. С. 324.

[4] И. Б. Краснюк, Б. Л. Ревзин. ИФЖ. 1994. Т. 67. № 3, 4. С. 167.

[5] И. Б. Краснюк. ИФЖ. 1994. Т. 67. № 1, 2. С. 162.

[6] И. Б. Краснюк, Т. Т. Рискиев. Оптика и спектроскопия. 1993. Т. 75. Вып. 3. С. 517.

[7] И. Б. Краснюк, Т. Т. Рискиев, Б. Л. Ревзин. Узб. физ. журн. 1993. № 4. С. 5.

[8] И. Б. Краснюк, Т. Т. Рискиев, А. А. Стрельцов. Узб. физ. журн. 1994. № 1. С. 50.

[9] I. B. Krasniuk. The systems with concurrens interaction oscillations of relaxational type. Int. Conf. of Bifurcations and Chaos. Crimea, 1994.

[10] И. Б. Краснюк. Об одном обобщении нелинейной гиперболической краевой задачи на плоскости. В кн.: Дифференциально-разностные уравнения и задачи математической физики. Киев: Институт математики, 1984. С. 38.

[11] М. Кац. Несколько вероятностных задач физики и математики. М.: Мир, 1995.

[12] В. П. Маслов, В. Г. Данилов, К. А. Волосов. Математическое моделирование процессов массотеплопереноса. Эволюция диссипативных структур. М.: Наука, 1987.

[13] Н. Н. Боголюбов, Ю. А. Митропольский. Асимптотические методы в теории нелинейных колебаний. М.: Наука, 1974.

[14] В. П. Маслов. Комплексный метод ВКБ в линейных уравнениях. М.: Наука, 1977.

[15] Г. Вентцель. Введение в квантовую теорию волновых полей. М.: Гостехиздат, 1947.

[16] В. К. Годунов, А. А. Султагазин. УМН. 1971. Т. 26. № 3.

[17] B. В. Веденяпин. О теореме существования в целом решения задачи Коши некоторых гиперболических нелинейных систем уравнений в частных производных (с приложениями к дискретным моделям уравнения Больцмана): Препринт ИПМ РАН № 42, 1973.

[18] И. Б. Краснюк. Докл. АН УССР. Сер. А. 1989. № 6. С. 20.

[19] А. Н. Шарковский, И. Б. Краснюк, Ю. Л. Майстренко. Докл. АН УССР. 1984. № 12. C. 27.

Поступила в редакцию 10.IV.1996 г., после доработки 13.IX.1996 г.

\section{B. Krasniuk \\ NONLINEAR BOUNDARY PROBLEM FOR BOLTZMAN'S EQUATIONS: IMPULSE SOLUTIONS AND THEIR BIFURCATIONS}

The statement of the nonlinear boundary conditions problem for the Boltzmann kinetic equation is considered. On this basis a possibility to form in the gases asymptoticaly periodic oscillations of the relaxation or preturbulent types is discussed. 